\title{
Imaging Impulsivity in Parkinson's Disease and the Contribution of the Subthalamic Nucleus
}

\author{
Nicola Ray, ${ }^{1,2}$ Francesca Antonelli, ${ }^{1,2}$ and Antonio P. Strafella ${ }^{1,2}$ \\ ${ }^{1}$ Toronto Western Research Institute and Hospital, UHN, University of Toronto, Toronto, ON, Canada M5T 2S8 \\ ${ }^{2}$ PET Imaging Centre, Centre for Addiction and Mental Health, University of Toronto, Toronto, ON, Canada M5T 2S8
}

Correspondence should be addressed to Antonio P. Strafella, astrafel@uhnres.utoronto.ca

Received 29 October 2010; Accepted 20 April 2011

Academic Editor: Irena Rektorova

Copyright (c) 2011 Nicola Ray et al. This is an open access article distributed under the Creative Commons Attribution License, which permits unrestricted use, distribution, and reproduction in any medium, provided the original work is properly cited.

\begin{abstract}
Taking risks is a natural human response, but, in some, risk taking is compulsive and may be detrimental. The subthalamic nucleus (STN) is thought to play a large role in our ability to inhibit responses. Differences between individuals' ability to inhibit inappropriate responses may underlie both the normal variation in trait impulsivity in the healthy population, as well as the pathological compulsions experienced by those with impulse control disorders (ICDs). Thus, we review the role of the STN in response inhibition, with a particular focus on studies employing imaging methodology. We also review the latest evidence that disruption of the function of the STN by deep brain stimulation in patients with Parkinson's disease can increase impulsivity.
\end{abstract}

\section{Introduction}

Some of us are more impulsive than others, more likely to take risks and suffer the consequences or reap the rewards. Taking risks is a natural human response; we would not be flying through the skies and sailing across oceans without it. However, in some, risk taking is compulsive and damaging. Risk taking that is detrimental may take the form of, for example, pathological gambling, where sufferers are unable to withhold from placing bets even after losses have become unmanageable. Other examples include hyper-sexuality, compulsive eating, and compulsive shopping, all of which can be described as impulse control disorders (ICDs). ICDs have been likened phenomenologically, epidemiologically, and neurologically with substance addiction, and may therefore be thought of as behavioral addictions [1].

In individuals with substance or behavioral addictions, impulsiveness is a common personality trait and may be associated with a vulnerability for addiction [2]. This vulnerability will likely have roots in individuals' socioeconomic background, and be heavily influenced by behaviors prevalent within ones family and peer group. By cause or consequence, vulnerability to addiction is also associated with particular patterns of brain chemistry, most notably in the dopaminergic systems of the basal ganglia [3]. However, these chemical differences may not be pathological, but only normal deviation. For example, in individuals without addiction, the same chemical differences are associated with the impulsive personality trait [4]. Thus, when such chemistry is combined with a lifestyle in which addictive stimuli are present and often available, addiction is more likely to ensue.

It may not be possible to "treat" vulnerability for addiction if it is based in the normal variation of brain transmitter systems. Instead, how those vulnerabilities affect how a person is able to control ones responses may be targeted. Response inhibition, and deficits thereof, is a subject of increased debate in the neuroscientific community. In particular, how the frontal cortex and basal ganglia, most notably the subthalamic nucleus (STN), act to allow the fast and effective inhibition of inappropriate responses is an area of research currently revealing some interesting findings.

The STN is one node within multiple, segregated corticobasal ganglia-thalamo-cortical circuits subserving motor, cognitive, and affective functions. Dorsal and lateral regions of the STN connect with sensorimotor areas of the basal ganglia and thalamus, premotor, and motor cortical areas. On the other hand, ventro-medial STN regions communicate with higher-order cortical regions subserving response inhibition, such as the anterior cingulate cortex (ACC) and inferior frontal gyrus (IFG) [5-7]. 
In this paper, we provide an overview of the current knowledge of how the STN is involved in response inhibition, and therefore how disruption to its function, for example, during deep brain stimulation in Parkinson's patients, might contribute to pathologically impulsive behaviors.

\section{Imaging of the STN during Impulsivity Tasks}

Impulsivity in the motor domain can be measured via the stop-signal paradigm [8] and the go/no-go paradigm, in which a "stop" response is pitted against a "go" response. In a typical experiment, choice reaction times to an imperative go-stimulus are measured and are followed on a proportion of trials, after a variable delay (or no delay in the go/nogo tasks), by a stop-stimulus instructing participants to withhold their response. Individuals with behavioral and chemical addictions have been found to perform poorly on tasks such as these [9-11]. Thus, as mentioned above, impulsive behavior may be rooted in impaired response inhibition.

In healthy controls response inhibition, as measured in stop-signal and go/no-go tasks, is found to be dependent on activity in the right inferior frontal cortex (IFC) [12$14]$, and, more recently, the subthalamic nucleus $[12,15$, 16]. It has been proposed that, during stopping, the right IFC sends a signal via the "hyperdirect" pathway [17] to the right STN. The STN then expedites the inhibition of activity in thalamocortical loops related to the action to be inhibited [12]. In the later paper, the authors found support for this hypothesis in that there was a blood oxygen leveldependent (BOLD) response in the STN to stop-signals. Further, participants with faster reaction times to the stopsignals showed larger responses than those with shorter reaction times in both the IFC and the STN, and there was a significant correlation between stop-signal reaction times and BOLD responses in these areas across subjects. Importantly, these authors confirmed that activity during the stop-signals was located in the STN using high-resolution structural and functional imaging in a second experiment within the same paper [12].

Alternatively, Li et al. [16], also measuring BOLD responses using fMRI during a stop-signal task in healthy participants, separated stop-trials in which inhibition was successful from those in which the participant was unable to withhold from performing the go-response. It was found that STN activity is the greatest during unsuccessful compared with successful inhibition. It was also greatest in those individuals who took longer to respond, or rather, not respond, to a stop-signal. These findings suggest that the role of the STN in response inhibition may be to process attentional aspects of the stop-signal and/or monitor performance, and not to act as a pathway for faster inhibition of the go-response. Supporting an attentional role, lesions of the STN produce attentional deficits in rats [18]. That said, activity in the STN, recorded in PD patients after deep brain stimulation surgery, during stopping in a go/no-task [19], has been shown to increase within frequency bands known to be associated with a lack of movement in the negative symptoms of PD [20-23].
Clearly, whatever the STNs' role in response inhibition, it must work in tandem with cortical and other brain areas in order that the conditions of stopping can be incorporated into the stop response. For example, Aron et al. [15] used diffusion-weighted imaging (DWI) tractography to show that the IFC and the STN region are connected. They were also able to report that both the inferior frontal cortex (IFC) and the STN region are connected with the presupplementary motor area (preSMA). The authors then found, using functional magnetic resonance imaging (fMRI) during a conditional stop-signal paradigm to study the neural control of slowing of go-responses in the presence of conflict, that the preSMA, IFC, and STN region were activated more when conflict-induced slowing was the greatest. Further, as discussed in more detail below, the anterior cingulate and its communication with the STN has been implicated in processes related to response inhibition.

\section{Imaging and Behavioral Studies on DBS of the STN during Measures of Response Inhibition}

Deep brain stimulation of the STN improves the motor symptoms of Parkinson's disease. It has also allowed us to, theoretically, interrupt function of the STN for experimental means. In the next section, we discus how DBS of the STN might increase impulsiveness in PD patients; here, we discuss how this impulsiveness might be brought about via interrupted response inhibition.

A prominent paper on the effects of STN DBS on impulsivity compared decision making under conflict after DBS and after dopamine replacement therapy in PD patients [24]. It was found that each of these interventions had their own effect on impulsiveness during the task. While medication was shown to impair learning from negative outcomes, DBS was shown to impair patients' ability to slow down when faced with conflict, an ability that serves to allow us time to settle on the decision most likely to yield positive outcomes. Thus, both STN DBS and medication may incur impulsive behavior, but do so via different routes.

It must be noted, however, that measuring response inhibition while patients receive DBS has yielded conflicting results. Response inhibition is shown to improve, remain unaffected, or worsen during DBS [25-30]. Such results may be due to a marginal role played by the STN in response inhibition. However, Ray et al. [31], Wylie et al. [32], and Hershey et al. [33] provide possible explanations for these discrepancies. Ray et al. describe deficits in inhibitory control only when improvements on the task due to improved motor control more generally are controlled for. Wylie et al. describe dissociabletemporal effects of STN DBS in that stimulation increased impulsive responding, but also improved the proficiency with which inhibitory control was engaged. Finally, Hershey et al. discovered differences in the effect of STN DBS on inhibitory control depending on whether the ventral or dorsal STN is stimulated.

That said, imaging the brain during response inhibition tasks has revealed interesting results. For example, 
Cambell et al. [29] used PET and [(15)O]-water to measure STN DBS-induced STN DBS-induced variability in cognitive performance, focusing on working memory and response inhibition. They found that STN DBS-induced blood flow changes in the dorsolateral prefrontal cortex, that correlated with a change in working memory performance. On the other hand, STN DBS caused blood flow changes in the anterior cingulate cortex, that correlated with a change in response inhibition. This suggests that stimulation of the STN may induce changes in the cortical (ACC) control of response inhibition, and the more it does so in individual patients, the greater the impairment in response inhibitions.

A later PET and [(15)O]-water measured blood flow during a Go/NoGo and a control (Go) task to study response inhibition deficits associated with STN-DBS [30]. They found that STN DBS improved motor scores on the Unified Parkinson Disease Rating Scale, but impaired response inhibition, measured as a greater number of errors during NoGo trials. The PET results revealed that changes on the task were accompanied by reduced activation in the left PMC, pre-SMA, dorsal ACC, and IFC, areas thought to subserve retroactive response inhibition in which a stimulus to stop must be processed and acted upon in order that inhibition is successful. The authors also found reduced activation in the precuneus and left inferior parietal cortex, which they argue play an important role, via interaction with the medial prefrontal cortex, in proactive (preparing to stop) inhibitory processes.

Given the data above, it seems that the STN plays a large role in response inhibition via its connections within the basal ganglia as well as areas of cortex involved in the cognitive aspects of controlling actions, such as the ACC, IFC, the medial prefrontal cortex, and dorsolateral prefrontal cortex. Thus, it is interesting to note that altered activity in some of these areas is found to be associated with the development ICDs in both the general population [34] and in PD patients who develop an ICD subsequent to dopamine replacement medications [35].

\section{Does DBS of the STN Induce Impulsive Control Disorders?}

Given the research discussed above, the question of whether DBS of the STN can alter patients' tendency to be impulsive one way or the other must be asked. Up to now, several conflicting reports have stressed a direct correlation between STN-DBS and impulse control disorders (ICDs). In some reports, patients with preoperative ICDs significantly improved after surgery [36-38]. However, in all cases, the phenomena was accompanied by a significant reduction of dopaminergic therapy. It was argued, therefore, that this improvement may be explained by pharmacological therapy reduction rather than the introduction of STN DBS [37]. In other reports, however, the development of an ICD was reported to occur secondary to STN-DBS [38-43]. In these cases, the disorder appeared a few months after surgery and was transient, resolving within a year of its development $[42,43]$. Some authors suggest that psychosocial factors, such as male gender and patient compliance, might influence the potential for the development of an ICD after STN DBS [38].

A behavior closely related to ICDs is the development of excessive or inappropriate levodopa use, or drug hording, described as a symptom of hedonistic homeostatic deregulation by Giovannoni et al. [44]. This condition has been described postoperatively in one patient treated with STN DBS [44]; however, most of the time this behavior develops preoperatively $[43,45]$.

More recently, a few prospective, but more frequently retrospective, trials were conducted in order to better understand the incidence and prevalence of ICDs in DBS STN patients and to confirm a possible direct role of STN surgery in inducing impulsive behaviors. However, to date, a scarcity of data precludes our ability to make any firm conclusions regarding the causal role of STN DBS in the development of ICDs. That said, a recent cross-sectional study assessed the degree of trait impulsivity in a group of 16 PD patients with STN DBS and a group of 37 PD patients without DBS, matched for levodopa equivalent doses (LEDD). The evaluation, performed only postoperatively, observed ICDs in $19 \%$ of DBS PD patients (3/16) and in $8 \%(3 / 37)$ of the non-DBS PD patients. The authors concluded that STN DBS might induce ICDs in PD. However, such inferences are limited without a preoperative evaluation [46]. Lim et al. [38], following a series of 21 patients with ICDs and dopamine disregulation syndrome (DDS) at some stage after the onset of $\mathrm{PD}$, found, from 17 patients with preoperative problems, 10 who experienced a worsening or no improvement of their ICDs after STN DBS, 5 who improved or resolved and 3 who developed ex novo DDS and ICDs after the surgery. Further, the authors noted that the behavioral response to STN DBS might be predicted by the vigilance of the physician, the motor outcome and patient compliance. Finally, in a recent study on punding (the appearance of repetitive, complex, and stereotypical behaviors), using a patient and relative completed survey of 24 consecutive PD patients with STN DBS [47], 20.8\% (5/24 in a group of PD DBS) were identified as punders, a higher percentage than the $14 \%(17 / 123$ in a group of PD) previously found by Evans in a subgroup of PD on dopaminergic therapy, or 1,4\% (4/291 in a group of PD) recently identified by Miyasaki in the same group. The higher ratio might be due to differences in the population studied.

While the studies above seem to suggest more impulsive behavior after STN DBS, Houeto et al., in a retrospective study on 24 patients, using a rating scale of personality change, found no differences in impulsivity pre- versus postoperatively in STN DBS PD patients [43].

As discussed above, many studies have attempted to test some of the underlying constructs of impulsive disorders, that is, using tasks that measuring motor and cognitive impulsivity. The majority of these studies have shown impairment while STN stimulation is on versus off $[25,27$, 47-50]. However, Pillon et al. [50] found no differences on a gambling task in patients with STN DBS turned on versus off.

In conclusion, although these studies suggest overall that impulsive disorders may be related to STN DBS, we would like to stress the need for larger, prospective, controlled 
trials to better understand the role of DBS in inducing ICDs. Recently, it has been proposed that impulsive patients might be at greater risk for postoperative suicide attempts [51]; however so far, there is no evidence supporting the presence of preoperative ICDs as a criterion of exclusion for surgery [51]. Neither do we have much inclination how to manage impulsive disorders that may appear postoperatively. In the case of ICDs occuring preoperatively, Voon et al. [52] have suggested that the relationship between ICDs and medication should be assessed before proceeding with surgery. If the problem persists, surgery should only be an option after an evaluation of the patients' cognitive status, their support, the intensity of the disorder and the patients' capacity for behavioral control. In the case of postoperative ICDs, Voon suggests medication reduction should be tried before considering a decrease in the intensity of the stimulation. Finally, she suggests that patients may benefit from the addition of an atypical antipsychotic to their regular medication, and from a multidisciplinary approach to managing the problem.

\section{References}

[1] M. N. Potenza, "The neurobiology of pathological gambling and drug addiction: an overview and new findings," Philosophical Transactions of the Royal Society of London. Series B, vol. 363, no. 1507, pp. 3181-3189, 2008.

[2] C. Esposito-Smythers, A. Spirito, C. Rizzo, J. E. McGeary, and V.S. Knopik, "Associations of the DRD2 TaqIA polymorphism with impulsivity and substance use: preliminary results from a clinical sample of adolescents," Pharmacology, Biochemistry and Behavior, vol. 93, no. 3, pp. 306-312, 2009.

[3] T. D. L. Steeves, J. Miyasaki, M. Zurowski et al., "Increased striatal dopamine release in Parkinsonian patients with pathological gambling: a [11C] raclopride PET study," Brain, vol. 132, no. 5, pp. 1376-1385, 2009.

[4] J. W. Buckholtz, M. T. Treadway, R. L. Cowan et al., "Dopaminergic network differences in human impulsivity," Science, vol. 329, no. 5991, p. 532, 2010.

[5] A. Parent and L. N. Hazrati, "Functional anatomy of the basal ganglia. II. The place of subthalamic nucleus and external pallidum in basal ganglia circuitry," Brain Research Reviews, vol. 20, no. 1, pp. 128-154, 1995.

[6] C. Hamani, J. A. Saint-Cyr, J. Fraser, M. Kaplitt, and A. M. Lozano, "The subthalamic nucleus in the context of movement disorders," Brain, vol. 127, no. 1, pp. 4-20, 2004.

[7] Y. Temel, A. Blokland, H. W. M. Steinbusch, and V. VisserVandewalle, "The functional role of the subthalamic nucleus in cognitive and limbic circuits," Progress in Neurobiology, vol. 76, no. 6, pp. 393-413, 2005.

[8] G. D. Logan, W. B. Cowan, and K. A. Davis, "On the ability to inhibit simple and choice reaction time responses: a model and a method," Journal of Experimental Psychology: Human Perception and Performance, vol. 10, no. 2, pp. 276-291, 1984.

[9] A. J. Lawrence, J. Luty, N. A. Bogdan, B. J. Sahakian, and L. Clark, "Impulsivity and response inhibition in alcohol dependence and problem gambling," Psychopharmacology, vol. 207, no. 1, pp. 163-172, 2009.

[10] A. E. Goudriaan, J. Oosterlaan, E. De Beurs, and W. Van Den Brink, "The role of self-reported impulsivity and reward sensitivity versus neurocognitive measures of disinhibition and decision-making in the prediction of relapse in pathological gamblers," Psychological Medicine, vol. 38, no. 1, pp. 41-50, 2008.

[11] A. Verdejo-García, A. Bechara, E. C. Recknor, and M. PérezGarcía, "Executive dysfunction in substance dependent individuals during drug use and abstinence: an examination of the behavioral, cognitive and emotional correlates of addiction," Journal of the International Neuropsychological Society, vol. 12, no. 3, pp. 405-415, 2006.

[12] A. R. Aron and R. A. Poldrack, "Cortical and subcortical contributions to stop signal response inhibition: role of the subthalamic nucleus," The Journal of Neuroscience, vol. 26, no. 9, pp. 2424-2433, 2006.

[13] B. U. Forstmann, S. Jahfari, H. S. Scholte, U. Wolfensteller, W. P. M. van den Wildenberg, and K. R. Ridderinkhof, "Function and structure of the right inferior frontal cortex predict individual differences in response inhibition: a model-based approach," The Journal of Neuroscience, vol. 28, no. 39, pp. 9790-9796, 2008.

[14] G. Xue, A. R. Aron, and R. A. Poldrack, "Common neural substrates for inhibition of spoken and manual responses," Cerebral Cortex, vol. 18, no. 8, pp. 1923-1932, 2008.

[15] A. R. Aron, T. E. Behrens, S. Smith, M. J. Frank, and R. A. Poldrack, "Triangulating a cognitive control network using diffusion-weighted magnetic resonance imaging (MRI) and functional MRI," The Journal of Neuroscience, vol. 27, no. 14, pp. 3743-3752, 2007.

[16] C. R. Li, P. Yan, R. Sinha, and T. W. Lee, "Subcortical processes of motor response inhibition during a stop signal task," NeuroImage, vol. 41, no. 4, pp. 1352-1363, 2008.

[17] A. Nambu, H. Tokuno, and M. Takada, "Functional significance of the cortico-subthalamo-pallidal 'hyperdirect' pathway," Neuroscience Research, vol. 43, no. 2, pp. 111-117, 2002.

[18] C. Baunez and T. W. Robbins, "Effects of dopamine depletion of the dorsal striatum and further interaction with subthalamic nucleus lesions in an attentional task in the rat," Neuroscience, vol. 92, no. 4, pp. 1343-1356, 1999.

[19] M. Cassidy, P. Mazzone, A. Oliviero et al., "Movement-related changes in synchronization in the human basal ganglia," Brain, vol. 125, no. 6, pp. 1235-1246, 2002.

[20] A. A. Kühn, A. Kupsch, G. H. Schneider, and P. Brown, "Reduction in subthalamic $8-35 \mathrm{~Hz}$ oscillatory activity correlates with clinical improvement in Parkinson's disease," The European Journal of Neuroscience, vol. 23, no. 7, pp. 19561960, 2006.

[21] N. J. Ray, N. Jenkinson, S. Wang et al., "Local field potential beta activity in the subthalamic nucleus of patients with Parkinson's disease is associated with improvements in bradykinesia after dopamine and deep brain stimulation," Experimental Neurology, vol. 213, no. 1, pp. 108-113, 2008.

[22] M. Weinberger, W. D. Hutchison, and J. O. Dostrovsky, "Pathological subthalamic nucleus oscillations in PD: can they be the cause of bradykinesia and akinesia?" Experimental Neurology, vol. 219, no. 1, pp. 58-61, 2009.

[23] A. Eusebio and P. Brown, "Synchronisation in the beta frequency-band - the bad boy of parkinsonism or an innocent bystander?" Experimental Neurology, vol. 217, no. 1, pp. 1-3, 2009.

[24] M. J. Frank, J. Samanta, A. A. Moustafa, and S. J. Sherman, "Hold your horses: impulsivity, deep brain stimulation, and medication in Parkinsonism," Science, vol. 318, no. 5854, pp. 1309-1312, 2007.

[25] M. Jahanshahi, C. M. Ardouin, R. G. Brown et al., "The impact of deep brain stimulation on executive function in Parkinson's disease," Brain, vol. 123, no. 6, pp. 1142-1154, 2000. 
[26] T. Hershey, F. J. Revilla, A. Wernle, P. S. Gibson, J. L. Dowling, and J. S. Perlmutter, "Stimulation of STN impairs aspects of cognitive control in PD," Neurology, vol. 62, no. 7, pp. 11101114, 2004.

[27] K. Witt, U. Pulkowski, J. Herzog et al., "Deep brain stimulation of the subthalamic nucleus improves cognitive flexibility but impairs response inhibition in Parkinson disease," Archives of Neurology, vol. 61, no. 5, pp. 697-700, 2004.

[28] S. Thobois, G. R. Hotton, S. Pinto et al., "STN stimulation alters pallidal-frontal coupling during response selection under competition," Journal of Cerebral Blood Flow and Metabolism, vol. 27, no. 6, pp. 1173-1184, 2007.

[29] M. C. Campbell, M. Karimi, P. M. Weaver et al., "Neural correlates of STN DBS-induced cognitive variability in Parkinson disease," Neuropsychologia, vol. 46, no. 13, pp. 3162-3169, 2008.

[30] B. Ballanger, T. van Eimeren, E. Moro et al., "Stimulation of the subthalamic nucleus and impulsivity: release your horses," Annals of Neurology, vol. 66, no. 6, pp. 817-824, 2009.

[31] N. J. Ray, N. Jenkinson, J. Brittain et al., "The role of the subthalamic nucleus in response inhibition: evidence from deep brain stimulation for Parkinson's disease," Neuropsychologia, vol. 47, no. 13, pp. 2828-2834, 2009.

[32] S. A. Wylie, K. R. Ridderinkhof, W. J. Elias et al., "Subthalamic nucleus stimulation influences expression and suppression of impulsive behaviour in Parkinson's disease," Brain, vol. 133, no. 12, pp. 3611-3624, 2010.

[33] T. Hershey, M. C. Campbell, T. O. Videen et al., "Mapping GoNo-Go performance within the subthalamic nucleus region," Brain, vol. 133, no. 12, pp. 3625-3634, 2010.

[34] S. F. Miedl, T. Fehr, G. Meyer, and M. Herrmann, "Neurobiological correlates of problem gambling in a quasi-realistic blackjack scenario as revealed by fMRI," Psychiatry Research, vol. 181, no. 3, pp. 165-173, 2010.

[35] T. van Eimeren, G. Pellecchia, R. Cilia et al., "Drug-induced deactivation of inhibitory networks predicts pathological gambling in PD," Neurology, vol. 75, no. 19, pp. 1711-1716, 2010.

[36] T. Witjas, C. Baunez, J. M. Henry et al., "Addiction in Parkinson's disease: impact of subthalamic nucleus deep brain stimulation," Movement Disorders, vol. 20, no. 8, pp. 1052-1055, 2005.

[37] C. Ardouin, V. Voon, Y. Worbe et al., "Pathological gambling in Parkinson's disease improves on chronic subthalamic nucleus stimulation," Movement Disorders, vol. 21, no. 11, pp. 1941-1946, 2006.

[38] S. Lim, S. S. O’Sullivan, K. Kotschet et al., "Dopamine dysregulation syndrome, impulse control disorders and punding after deep brain stimulation surgery for Parkinson's disease," Journal of Clinical Neuroscience, vol. 16, no. 9, pp. 1148-1152, 2009.

[39] L. M. Romito, M. Raja, A. Daniele et al., "Transient mania with hypersexuality after surgery for high frequency stimulation of the subthalamic nucleus in Parkinson's disease," Movement Disorders, vol. 17, no. 6, pp. 1371-1374, 2002.

[40] P. Doshi and P. Bhargava, "Hypersexuality following subthalamic nucleus stimulation for Parkinson's disease," Neurology India, vol. 56, no. 4, pp. 474-476, 2008.

[41] H. M. M. Smeding, A. E. Goudriaan, E. M. J. Foncke, P. R. Schuurman, J. D. Speelman, and B. Schmand, "Pathological gambling after bilateral subthalamic nucleus stimulation in Parkinson disease," Journal of Neurology, Neurosurgery and Psychiatry, vol. 78, no. 5, pp. 517-519, 2007.
[42] M. Sensi, R. Eleopra, M. A. Cavallo et al., "Explosive-aggressive behavior related to bilateral subthalamic stimulation," Parkinsonism and Related Disorders, vol. 10, no. 4, pp. 247251, 2004.

[43] J. L. Houeto, V. Mesnage, L. Mallet et al., "Behavioural disorders, Parkinson's disease and subthalamic stimulation," Journal of Neurology, Neurosurgery and Psychiatry, vol. 72, no. 6, pp. 701-707, 2002.

[44] G. Giovannoni, J. D. O'Sullivan, K. Turner, A. J. Manson, and A. J. Lees, "Hedonistic homeostatic dysregulation in patients with Parkinson's disease on dopamine replacement therapies," Journal of Neurology, Neurosurgery and Psychiatry, vol. 68, no. 4, pp. 423-428, 2000.

[45] P. Krack, A. Batir, N. Van Blercom et al., "Five-year follow-up of bilateral stimulation of the subthalamic nucleus in advanced Parkinson's disease," The New England Journal of Medicine, vol. 349, no. 20, pp. 1925-1934, 2003.

[46] T. D. Hälbig, W. Tse, P. G. Frisina et al., "Subthalamic deep brain stimulation and impulse control in Parkinson's disease," European Journal of Neurology, vol. 16, no. 4, pp. 493-497, 2009.

[47] M. Alegret, C. Junqué, F. Valldeoriola et al., "Effects of bilateral subthalamic stimulation on cognitive function in Parkinson disease," Archives of Neurology, vol. 58, no. 8, pp. 1223-1227, 2001.

[48] K. Dujardin, L. Defebvre, P. Krystkowiak, S. Blond, and A. Destee, "Influence of chronic bilateral stimulation of the subthalamic nucleus on cognitive function in Parkinson's disease," Journal of Neurology, vol. 248, no. 7, pp. 603-611, 2001.

[49] R. Moretti, P. Torre, R. M. Antonello et al., "Neuropsychological changes after subthalamic nucleus stimulation: a 12 month follow-up in nine patients with Parkinson's disease," Parkinsonism and Related Disorders, vol. 10, no. 2, pp. 73-79, 2003.

[50] B. Pillon, C. Ardouin, P. Damier et al., "Neuropsychological changes between 'off' and 'on' STN or GPi stimulation in Parkinson's disease," Neurology, vol. 55, no. 3, pp. 411-418, 2000.

[51] V. Voon, P. Krack, A. E. Lang et al., "A multicentre study on suicide outcomes following subthalamic stimulation for Parkinson's disease," Brain, vol. 131, no. 10, pp. 2720-2728, 2008.

[52] V. Voon, C. Kubu, P. Krack, J. Houeto, and A. I. Tröster, "Deep brain stimulation: neuropsychological and neuropsychiatric issues," Movement Disorders, vol. 21, supplement 14, pp. S305S327, 2006. 


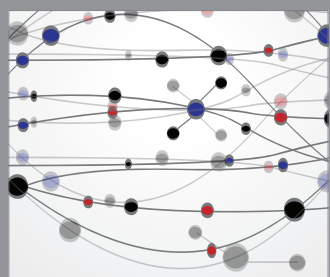

The Scientific World Journal
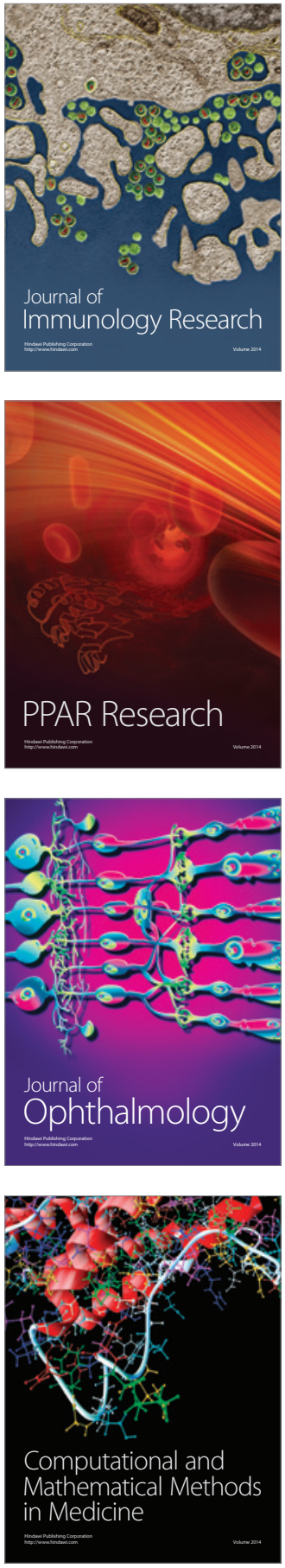

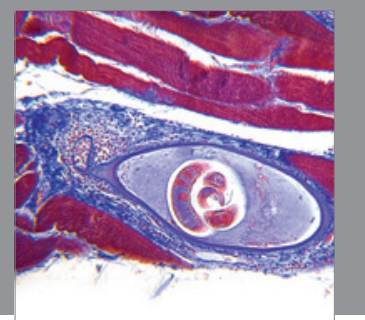

Gastroenterology

Research and Practice
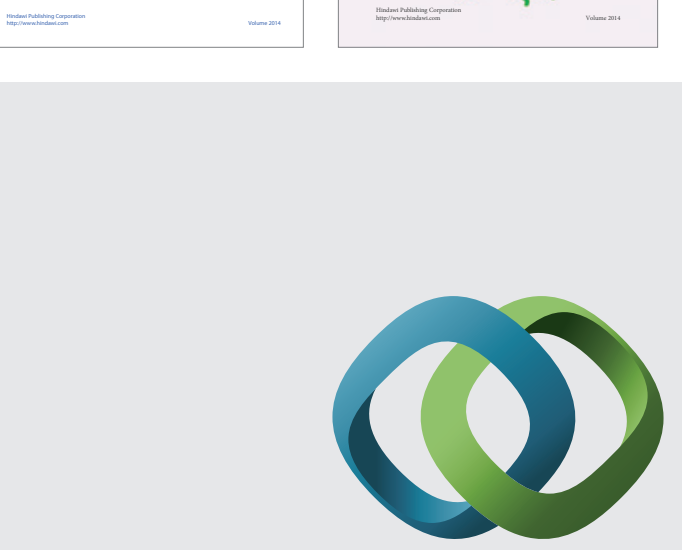

\section{Hindawi}

Submit your manuscripts at

http://www.hindawi.com
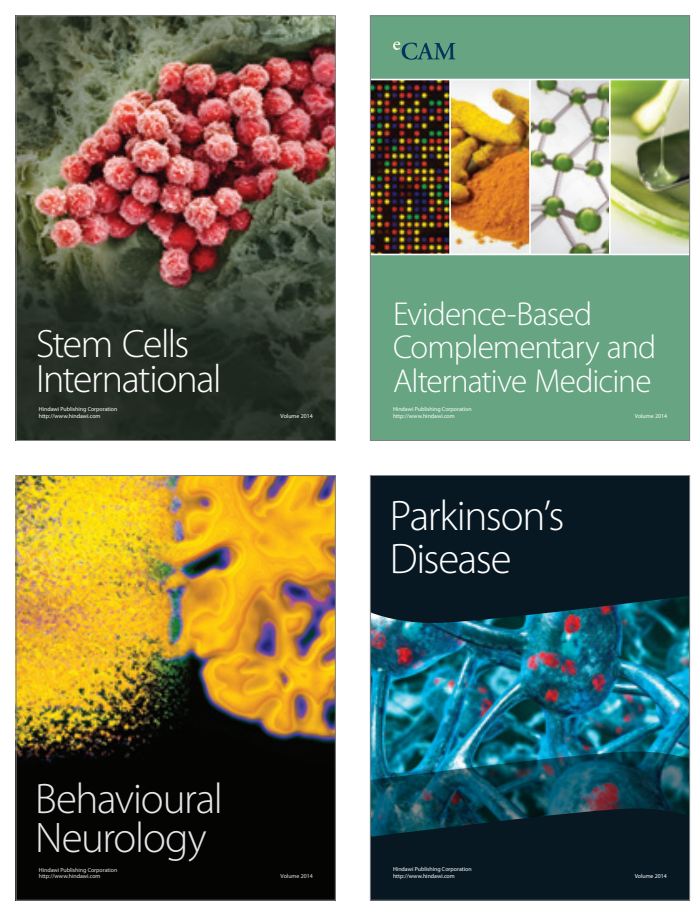

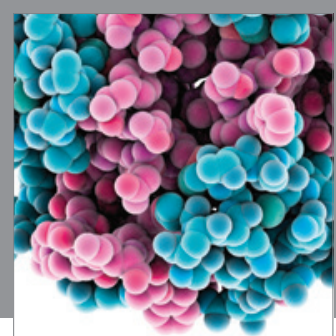

Journal of
Diabetes Research

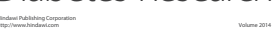

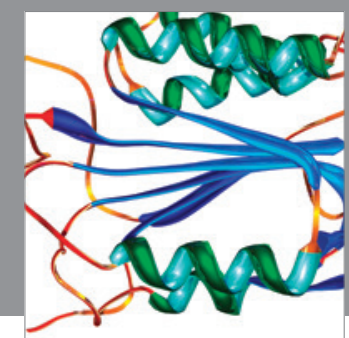

Disease Markers
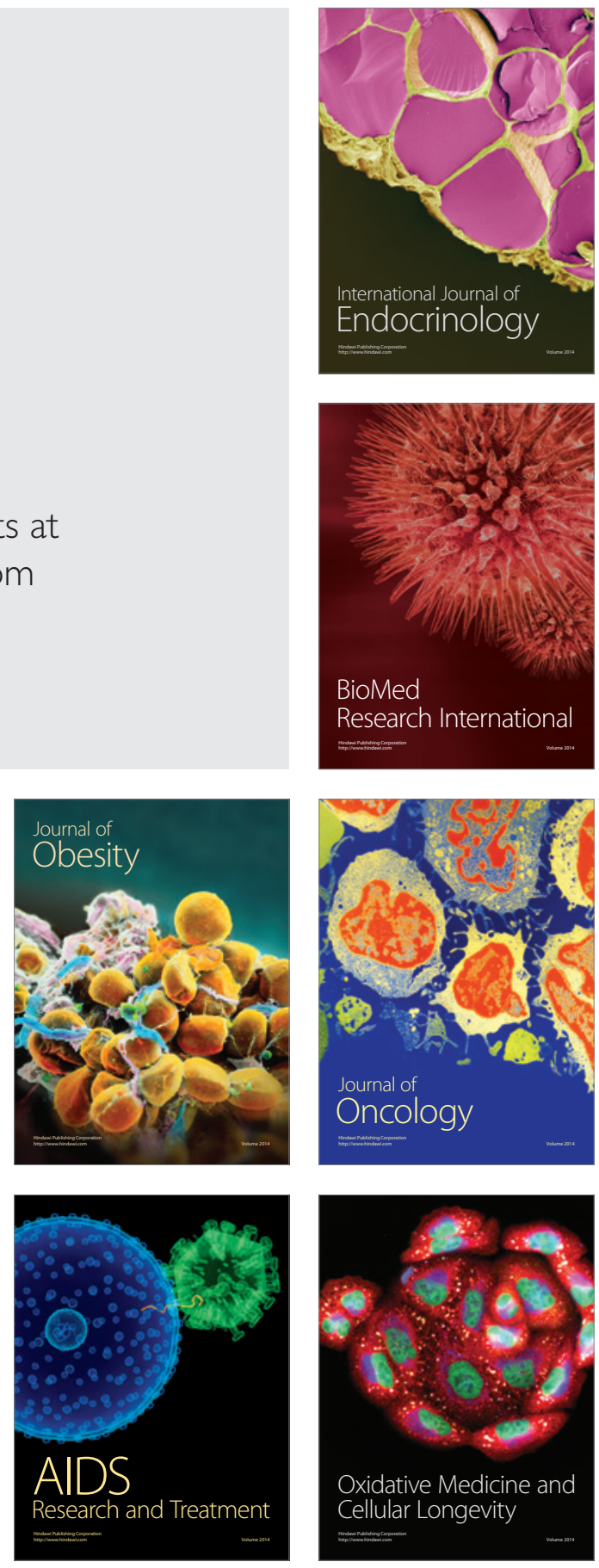